\title{
Article \\ The PCL Envelope Lack Sign (PELS) Is a Direct Arthroscopic Sign of Chronic Posterior Cruciate Ligament Insufficiency
}

\author{
Adrian Góralczyk ${ }^{1}$, Marcin Mostowy ${ }^{2}$, Michał Ebisz ${ }^{3}{ }^{1}$, Robert F. LaPrade ${ }^{4}$, Aleksandra Sibilska ${ }^{5}$, \\ Krzysztof Hermanowicz ${ }^{1}$, Marcin E. Domżalski ${ }^{2}$ and Konrad Malinowski ${ }^{3, *}$ \\ 1 ORTIM Orthopaedic Clinic, Mlynowa 17, 15-568 Bialystok, Poland; adriangoralczyk@wp.pl (A.G.); \\ herman10@go2.pl (K.H.) \\ 2 Orthopedic and Trauma Department, Veteran's Memorial Teaching Hospital in Lodz, \\ Medical University of Lodz, 90-549 Lodz, Poland; mostowymarcin1@gmail.com (M.M.); \\ marcin.domzalski@umed.lodz.pl (M.E.D.) \\ 3 Artromedical Orthopaedic Clinic, Antracytowa 1, 97-400 Belchatow, Poland; michal.ebisz@gmail.com \\ 4 Twin Cities Orthopedics, Edina, MN 55121, USA; laprademdphd@gmail.com \\ 5 Clinic of Orthopedics and Pediatric Orthopedics, Medical University of Lodz, 92-213 Lodz, Poland; \\ ola.sibilska.stud@gmail.com \\ * Correspondence: malwin8@wp.pl; Tel.: +48-509-812-212
}

check for updates

Citation: Góralczyk, A.; Mostowy, M.; Ebisz, M.; LaPrade, R.F.; Sibilska, A.; Hermanowicz, K.; Domżalski, M.E.; Malinowski, K. The PCL Envelope Lack Sign (PELS) Is a Direct Arthroscopic Sign of Chronic Posterior Cruciate Ligament Insufficiency. Appl. Sci. 2021, 11, 3608. https://doi.org/10.3390/app11083608

Academic Editor: Krzysztof Ficek

Received: 17 March 2021

Accepted: 15 April 2021

Published: 16 April 2021

Publisher's Note: MDPI stays neutral with regard to jurisdictional claims in published maps and institutional affiliations.

Copyright: (c) 2021 by the authors Licensee MDPI, Basel, Switzerland. This article is an open access article distributed under the terms and conditions of the Creative Commons Attribution (CC BY) license (https:// creativecommons.org/licenses/by/ $4.0 /)$.

\begin{abstract}
Purpose: To present the arthroscopic "PCL envelope lack sign" (PELS) and to calculate its diagnostic characteristics in chronic PCL insufficiency. Methods: Recordings of knee arthroscopies performed in a single clinic between April 2015 to March 2020 were retrospectively evaluated, searching for the "PCL envelope". It was defined as a "soft tissue cuff coursing around the PCL tibial attachment, visible with the arthroscope positioned between the PCL, medial femoral condyle and posterior horn of the medial meniscus at the level of its shiny white fibers". PELS was defined as "the PCL adhering to the proximal tibia adjacent to the medial meniscal posterior root attachment, inability to observe the normal space between the PCL and posterior tibia and no soft tissue cuff around the PCL tibial attachment". Inclusion criteria were possibility to evaluate the PELS presence on recordings. Patients who underwent PCL reconstruction were assigned to the study group. The rest of the patients were controls. Criteria to operate on symptomatic PCL patients were at least $5 \mathrm{~mm}$ of posterior instability in physical examination and at least 6 months post-injury. Results: Out of 614 available recordings, 592 patients ( 205 females, 387 males; mean age 45.2 years, SD = 14.36, range 14-81) were included: 38 in the study group and 554 in the control group. In the study group, PELS was positive in 36 of 38 cases (94.7\%). In the control group, PELS was negative in 554 PCL-efficient patients (100\%). Calculated PELS sensitivity was $94.7 \%$, specificity $100 \%$, positive predictive value $100 \%$, negative predictive value $99.6 \%$. The PELS was present significantly more often in PCL-insufficient patients, $p<0.001$. Conclusions: The PCL envelope lack sign was found to be a highly effective tool to arthroscopically confirm chronic PCL insufficiency, and should be considered a direct sign of chronic posterior knee instability.
\end{abstract}

Keywords: posterior cruciate ligament; chronic PCL insufficiency; arthroscopic sign; posterior knee arthroscopy

\section{Introduction}

Injuries of the posterior cruciate ligament (PCL) still remain as one of the most challenging pathologies to diagnose in knee surgery. Whereas acute PCL injuries can be easily detected by taking a thorough history, performing an adequate physical examination, and utilizing available imaging methods such as classic radiographs or magnetic resonance imaging (MRI) scans, significant difficulties may occur in chronic cases [1-3]. This is because a torn PCL usually heals, but often in a lax or attenuated position [1,4,5]. Thus, a patient with chronic posterior knee instability usually presents with uncharacteristic 
symptoms such as vague pain; stiffness; and problems in high knee flexion, descending inclines and stairs, and during deceleration movements $[1,3,5,6]$. Whereas both MRI and physical examination do not reach the expected sensitivity in chronic PCL tears, stress radiographs and arthroscopic evaluation of the ligament are the diagnostic tools of choice [3,6,7]. However, to date, there are no described direct arthroscopic signs of chronic PCL insufficiency. Available indirect signs, such as cartilage lesions in the medial compartment or patellofemoral joint, a "slack" anterior cruciate ligament (ACL) sign, or a posteromedial drive-through sign, are vulnerable to inaccuracy $[1,6,8]$. Therefore, direct arthroscopic sign of chronic PCL insufficiency could be very useful for arthroscopic surgeons.

The aim of this study is to present the arthroscopic "PCL envelope lack sign" (PELS) and to calculate its diagnostic characteristics in chronic PCL insufficiency. Our hypothesis was that PELS would be present more often in PCL-insufficient patients than in PCLefficient patients with good diagnostic characteristics.

\section{Materials and Methods}

Recordings of 614 knee arthroscopies performed in the (blinded for review) clinic between April 2015 to March 2020 were retrospectively evaluated for this study. A total of 38 patients (11 females, 27 males; mean age 37.0 years, $\mathrm{SD}=12.1$, range $17-53$ ) were operated on due to clinically significant posterior knee instability and were included in the study group. The indications for arthroscopy were symptomatic posterior knee instability with at least $5 \mathrm{~mm}$ of increased posterior tibia translation in physical examination and at least 6 months post-injury. Preoperative MRI was performed in all cases. However, MRI appearance of PCL in cases of chronic PCL injury was not used as inclusion criteria due to the fact that, as reported in the literature, sometimes PCL tears heal in unfunctional elongation even though their continuity is maintained $[1,4,5]$. The remaining 576 patients had an intact PCL (203 females, 373 males; mean age 43.7 years, SD = 14.9, range 14-81). The PCL was observed for the presence of an "PCL envelope" as a part of our standard arthroscopic inspection protocol. In cases of a suspected chronic PCL tear, we assessed for the presence of the PCL envelope lack sign (PELS). Inclusion criteria was the possibility to evaluate PELS presence on recordings. Within the group of patients with intact PCL, it was impossible to evaluate the presence of the PCL "envelope" in 22 cases (9 females, 13 males; mean age 63.9 years, $S D=11.0$, range $24-74$ ) due to large osteophytes on the medial femoral condyle or medial tibial eminence with no indications to extend the surgery by partial medial tibial eminence resection. Therefore, 554 PCL-intact patients (194 females, 360 males; mean age 42.9 years, $\mathrm{SD}=14.4$, range $14-81$ ) were included in the control group.

All recordings were analyzed by a single author with more than 20 years of experience in knee mini-invasive and arthroscopic surgery. In 2013, during arthroscopy in PCL-efficient knees, we started to observe an anatomic sign that we called a "PCL envelope", defined as the "soft tissue cuff coursing around the PCL tibial attachment, visible with arthroscope positioned between the PCL, medial femoral condyle and posterior horn of medial meniscus at the level of its shiny white fibers" (Figure 1). The shiny white fibers of the posterior horn of medial meniscus are important arthroscopic landmark in PCL surgeries [3,9]. To visualize the PCL envelope in a PCL-intact knee, we performed the arthroscopy through standard anterolateral and anteromedial portals using a $30^{\circ}$ arthroscope. After a thorough inspection of the whole joint, the PCL was assessed anteriorly and then the arthroscope was positioned between the PCL, MFC, and over the posterior horn of the medial meniscus $(\mathrm{MM})$ and inserted into the posteromedial recess using a trans-notch maneuver. Initially, visualization was directed medially toward the "ramp" of the MM. Then, the arthroscope was gradually withdrawn with rotational movements until the moment when the MFC and posterior root of MM and its shiny white fibers were observed. In this position, a soft tissue cuff coursing around the PCL tibial attachment could be observed (Figure 1a), which was the aforementioned PCL envelope. Next, the arthroscope was withdrawn a few millimeters more to visualize the PCL tibial attachment and the soft tissue envelope surrounding this attachment was observed (Figure 1b). 


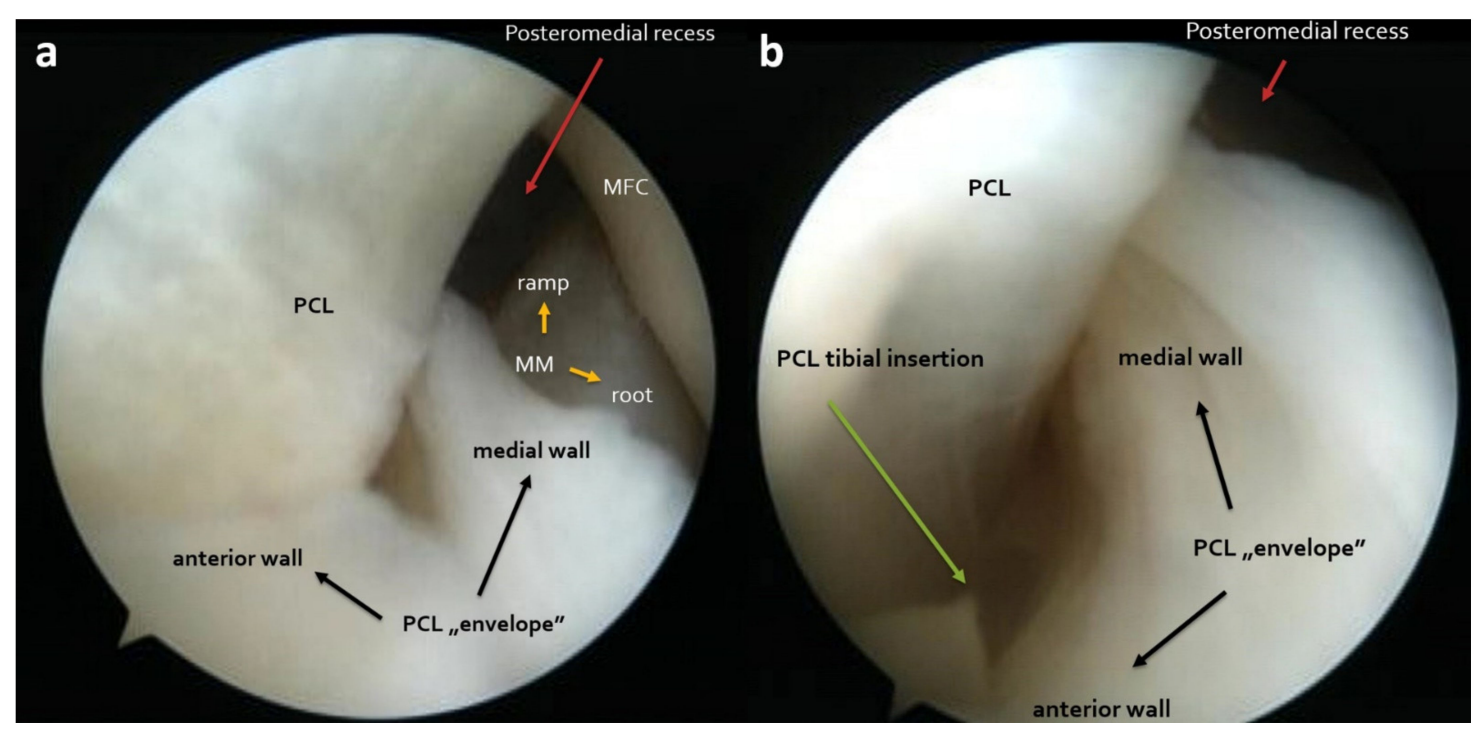

Figure 1. Arthroscopic view from an anterolateral viewing portal in a right knee joint. Trans-notch maneuver. (a) The natural soft tissue cuff surrounding the posterior cruciate ligament (PCL) tibial insertion, the so-called PCL "envelope", can be observed. (b) After withdrawing the arthroscope a few millimeters, we visualized the PCL tibial attachment with the surrounding envelope. MFC—-medial femoral condyle, MM-medial meniscus.

Then, we started to analyze this finding in knees with a suspected chronic PCL insufficiency, concluding that PCL envelope is something that usually cannot be observed in PCL-deficient knees. In the case of suspected chronic PCL insufficiency, when the arthroscope was positioned in the place where the PCL envelope could normally be visualized, it was not visible. Instead, phenomenon that we called the "PCL envelope lack sign" (PELS) could be seen, which we theorized could be a direct sign of chronic PCL insufficiency. The PELS was defined as "the PCL adhering to the proximal tibia adjacent to the medial meniscal posterior root attachment, inability to observe the normal space between the PCL and posterior tibia and no soft tissue cuff around the PCL tibial attachment" (Figure 2).

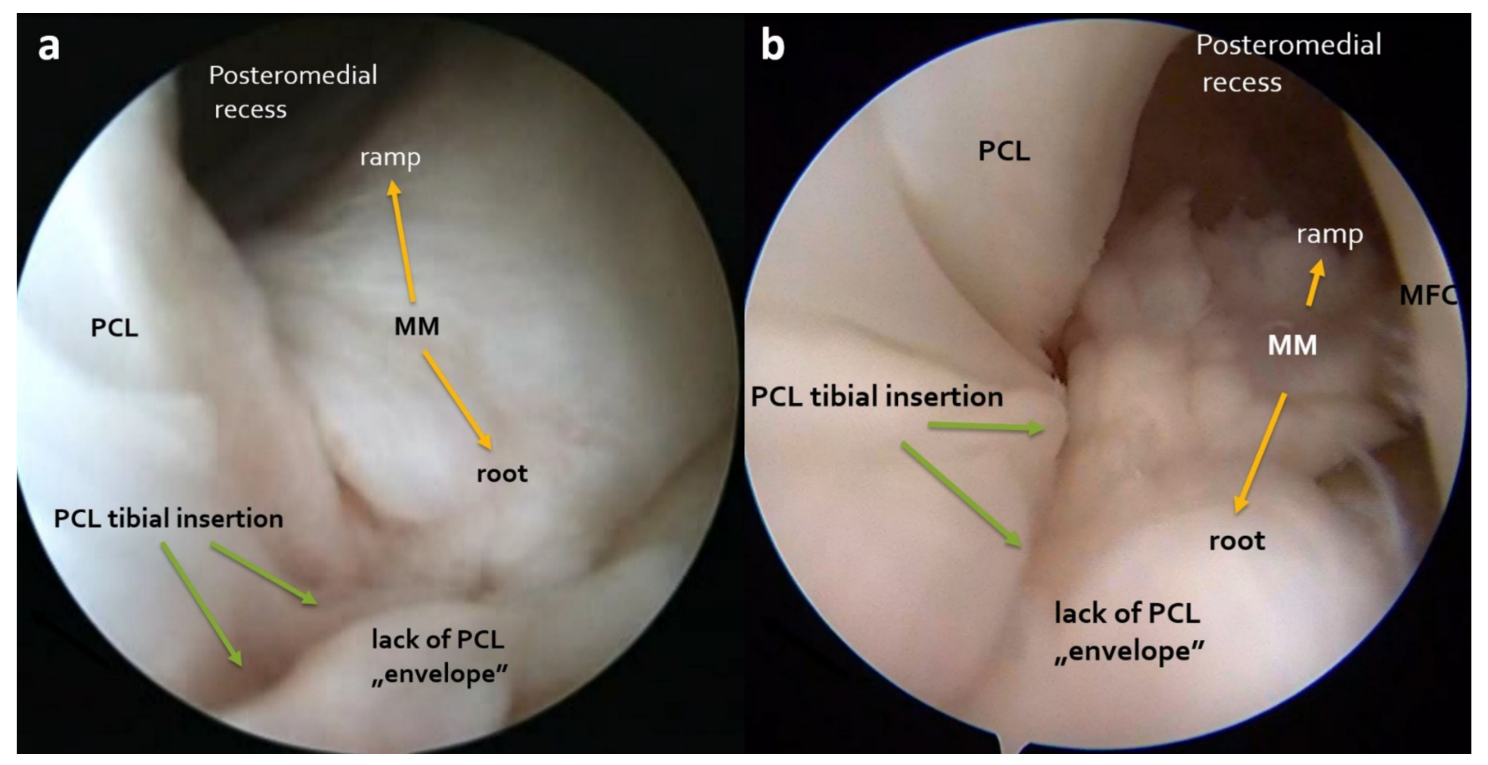

Figure 2. Arthroscopic view from an anterolateral viewing portal in a right knee joint. Trans-notch maneuver. (a) Positive PCL envelope lack sign. PCL tibial insertion starting at the level of joint line without a natural space between the PCL and tibia and medial meniscus (MM) posterior root adjacent to the PCL can be observed. (b) The same phenomenon seen from a different angle. MFC—-medial femoral condyle. 
After the evaluation of recordings, we analyzed results statistically with Statistica 13.1. software (StatSoft Poland). Sensitivity, specificity, positive predictive value, and negative predictive value of PELS were calculated. A double-tailed Fisher's exact test was used to assess whether the presence of PELS was significantly associated with chronic PCL insufficiency. In order to evaluate the difference of mean age between groups, we used the Mann-Whitney $U$ test due to the lack of normality of data distribution. Normality of distribution was assessed using the Shapiro-Wilk test. A chi-squared test with Yates correction was used to evaluate differences in sex distribution between groups. Significance was set at $p<0.05$ for all statistical tests.

This study was approved by the Institutional Ethics Committee of the authors' District Medical Chamber.

\section{Results}

There was no significant difference between the study group and control group as to the sex of the patient; however, the study group was significantly younger than the control group, with mean age 37.0 years vs. 42.9 years, respectively (Table 1 ).

Table 1. Demographics of study group and control group for the PCL envelope lack sign (PELS).

\begin{tabular}{cccc}
\hline & Study Group & Control Group & $p$-Value \\
\hline Mean age & 37.0 years, SD $=12.1$ & 42.9 years, SD $=14.4$ & $p=0.0224$ \\
\hline $\begin{array}{c}\text { Female to male ratio } \\
\text { (F:M, \% of females) }\end{array}$ & $\begin{array}{c}11 \mathrm{~F}: \text { : 27M (28.9\% of } \\
\text { females) }\end{array}$ & $\begin{array}{c}194 \mathrm{~F}: 360 \mathrm{M}(35.0 \% \text { of } \\
\text { females) }\end{array}$ & $p=0.5633$ \\
\hline SD-standard deviation, M-males, F-females. & &
\end{tabular}

In the study group, of the 38 cases with chronic PCL insufficiency, a positive PELS was observed in 36 cases (94.7\%). In the control group, the PELS was negative in 554 out of 554 PCL-intact patients (100\%). The PELS sensitivity was calculated to be $94.7 \%$, specificity $100 \%$, positive predictive value $100 \%$, and negative predictive value $99.6 \%$ (Table 2). The double-tailed Fisher's exact test revealed that the PELS was more often present in PCL-insufficient patients with $p<0.001$.

Table 2. Presence of the PCL envelope lack sign (PELS) in the study and control groups and its diagnostic characteristics.

\begin{tabular}{ccccc}
\hline & $\begin{array}{c}\text { Positive PELS } \\
\text { (Number, \%) }\end{array}$ & $\begin{array}{c}\text { Negative PELS } \\
\text { (Number, \%) }\end{array}$ & $\begin{array}{c}\text { Total Number of } \\
\text { Patients }\end{array}$ & Specificity = $100 \%$ \\
\hline PCL-efficient patients & 0 & 554 & 554 & Sensitivity $=94.7 \%$ \\
\hline PCL-insufficient patients & 36 & 2 & 38 & \\
\hline & PPV $=100 \%$ & NPV $=99.6 \%$ & & \\
\hline
\end{tabular}

PCL—posterior cruciate ligament, PELS—PCL envelope lack sign, PPV—positive predictive value, NPV—negative predictive value.

Four second-look knee postoperative arthroscopies were performed for other indications at least 2 months after the PCL reconstruction or repair. The PCL "envelope" was found to be restored in all four cases (Figure 3). 


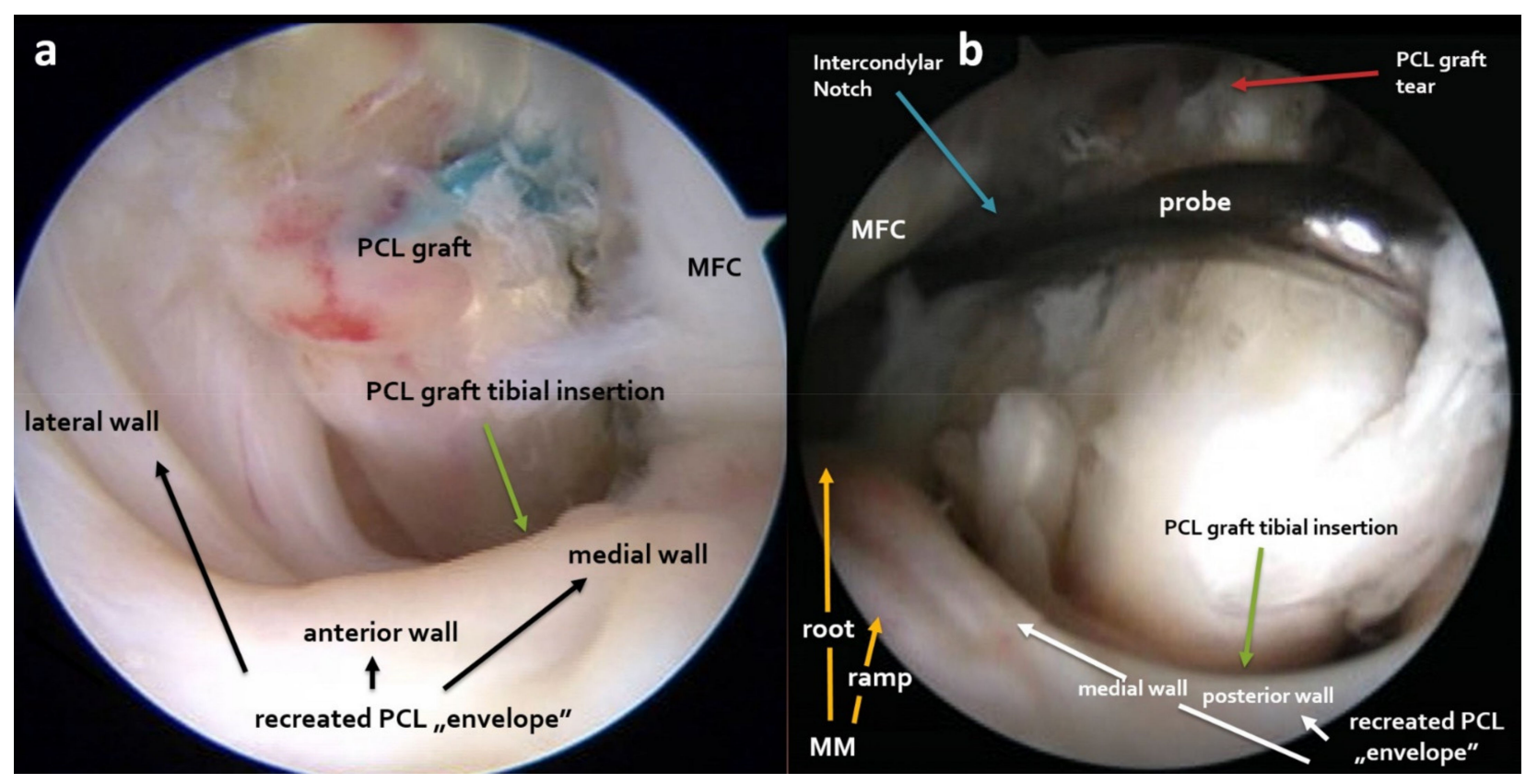

Figure 3. (a) Arthroscopic view from an anterolateral portal in a right knee joint after a PCL reconstruction. Trans-notch maneuver. The recreated PCL "envelope" can be observed between PCL graft and tibia. (b) Arthroscopic view from a posteromedial portal in a right knee joint after a PCL reconstruction. The recreated PCL envelope can be observed in the knee with a posttraumatic PCL graft tear. The probe is inserted through the anterolateral portal and intercondylar notch. MFC—-medial femoral condyle, MM—medial meniscus.

\section{Discussion}

The most important finding of this study was that the PCL envelope lack sign (PELS) was an effective arthroscopic diagnostic tool for confirmation of chronic PCL insufficiency. It is a direct sign of PCL deficiency, which can be observed on the ligament itself. Currently available arthroscopic tests to assess the integrity of the PCL, such as the "slack ACL sign", the posteromedial drive-through sign, and others, are only indirect signs and thus vulnerable to inaccuracy $[1,6,8]$. For example, the posteromedial drive-through sign, reported by Nha et al., in our opinion may be false-positive in the case of general joint laxity or medial knee instability [10]. It may also be false-negative in the presence of osteophytes or if the position of the anterolateral portal is very high and constrains one to change the angle of visualization, therefore demanding higher force to perform trans-notch maneuver [10]. Another indirect sign, cartilage lesions in the patellofemoral joint or medial compartment, observed in chronic PCL instability, in fact may be caused also by traumatic events, or even without any instabilities as a common pattern in OA development [11]. This is the reason why we searched for direct arthroscopic signs independent of other factors, which could be strong evidence for the orthopedic surgeon in directly evaluating for a PCL tear.

In the case of a positive PELS sign, (1) the PCL adheres to the proximal tibia adjacent to the medial meniscal posterior root attachment, (2) there is a loss of the natural free space between the PCL and posterior tibia, and (3) there is a loss of soft tissue cuff around the PCL attachment. While it is unclear why these three phenomena occur, possible explanation can be due to the posterior subluxation of tibia after PCL healing in elongation. In this situation, contact area between the PCL and posterior tibia may change and natural movements of PCL may alter, leading to PELS in two distinct mechanisms. The first mechanism of PELS formation can be pressing of elongated PCL against the posterior part of the tibia, which provides no natural free space anterior to the tibial part of the PCL. A second possible cause is formation of scar tissue between the soft tissue cuff and PCL substance, where there should be a free space in the healthy knee. It is also worth noting that natural PCL 
"envelope" may be recreated after PCL reconstruction, suggesting that PCL "envelope" may be necessary or is a natural effect of proper PCL functioning.

It is important to recognize that complete PCL tears reportedly take a median of 21 months from the index injury to the diagnosis for the requirement for operative treatment $[12,13]$. However, it has been reported that PCL reconstruction for posterior knee instability improves patient subjective functional scores and objectively restores knee stability $[4,7,14,15]$. This leads to the conclusion that a weak point in PCL injury management still remains in terms of making an accurate diagnosis $[12,14]$. Whereas physical examination and MRI may not be sufficient in chronic PCL insufficiency, stress radiographs and arthroscopy (indirect signs) remain a confirmatory tool for suspected PCL injury. As surgeons, we continuously pursue to offer optimal diagnostic solutions on the basis of more than just a single clinical test or diagnostic modality. We think that PELS can be a helpful confirmatory tool during arthroscopy to increase assurance that the preoperative diagnosis of the chronic PCL insufficiency is correct.

This study is recognized to have some limitations. The first limitation of the study is that the presence of PCL "envelope" was impossible to retrospectively assess in 22 out of 614 arthroscopy recordings. These patients had an intact PCL, and it is possible that some of them did not have a PCL "envelope". What is more, all patients were operated on in a single clinic and results remain to be repeated in multicenter trials. The next limitation is that presence of PELS was evaluated by a single surgeon. Even though that surgeon has more than 20 years of experience in knee mini-invasive and arthroscopic surgery, the single-evaluator study design did not allow us to calculate inter-rater reliability of PELS detection. Another limitation of the study is that although all PCL-insufficient patients were more than 6 months from PCL injury, we did not stratify cases as to the exact time from injury or other factors. However, with only two negative PELS in the group of 38 PCL-insufficient patients, our group was not big enough to stratify.

\section{Conclusions}

The PCL envelope lack sign was found to be a highly effective tool to arthroscopically confirm chronic PCL insufficiency and should be considered a direct sign of chronic posterior knee instability.

Author Contributions: Conceptualization, K.M.; methodology, A.G., M.M., M.E., R.F.L., A.S., K.H., M.E.D., and K.M.; formal analysis, M.M. and K.M.; resources, K.M.; data curation, K.M.; writingoriginal draft preparation, A.G., M.M., M.E., and A.S.; writing-review and editing, R.F.L., K.H., M.E.D., and K.M.; supervision, K.M.; project administration, K.M. All authors have read and agreed to the published version of the manuscript.

Funding: This research received no external funding.

Institutional Review Board Statement: The study was conducted according to the guidelines of the Declaration of Helsinki and approved by the Institutional Ethics Committee of District Medical Chamber in Lodz: protocol code K.B.-11/2020, date 3 June 2020.

Informed Consent Statement: Patient consent was waived due to the retrospective character of the study (analysis of recordings of performed arthroscopies).

Data Availability Statement: All data were reported in the manuscript.

Conflicts of Interest: The authors declare no conflict of interest.

\section{References}

1. Verhulst, F.V.; MacDonald, P. Diagnosing PCL Injuries: History, Physical Examination, Imaging Studies, Arthroscopic Evaluation. Sports Med. Arthrosc. 2020, 28, 2-7. [CrossRef] [PubMed]

2. Winkler, P.W.; Zsidai, B.; Wagala, N.N.; Hughes, J.D.; Horvath, A.; Senorski, E.H.; Samuelsson, K.; Musahl, V. Evolving evidence in the treatment of primary and recurrent posterior cruciate ligament injuries, part 1: Anatomy, biomechanics and diagnostics. Knee Surg. Sport Traumatol. Arthrosc. 2020. [CrossRef] [PubMed]

3. Chahla, J.; von Bormann, R.; Engebretsen, L.; LaPrade, R.F. Anatomic posterior cruciate ligament reconstruction: State of the Art. J. ISAKOS Jt. Disord. Orthop. Sport Med. 2016, 1, 292-302. [CrossRef] 
4. $\quad$ Laprade, C.M.; Civitarese, D.M.; Rasmussen, M.T.; Laprade, R.F. Emerging updates on the posterior cruciate ligament. Am. J. Sports Med. 2015, 43, 3077-3092. [CrossRef] [PubMed]

5. Vaquero-Picado, A.; Rodríguez-Merchán, E.C. Isolated posterior cruciate ligament tears: An update of management. EFORT Open Rev. 2017, 2, 89-96. [CrossRef] [PubMed]

6. Devitt, B.M.; Whelan, D.B. Clinical and Arthroscopic Evaluation of the Posterior-Cruciate-Ligament- Injured Knee. Posterior Cruciate Ligament Inj. 2015, 49-64. [CrossRef]

7. DePhillipo, N.N.; Cinque, M.E.; Godin, J.A.; Moatshe, G.; Chahla, J.; LaPrade, R.F. Posterior Tibial Translation Measurements on Magnetic Resonance Imaging Improve Diagnostic Sensitivity for Chronic Posterior Cruciate Ligament Injuries and Graft Tears. Am. J. Sports Med. 2018, 46, 341-347. [CrossRef] [PubMed]

8. Feng, H.; Song, G.Y.; Shen, J.W.; Zhang, H.; Wang, M. The "lateral gutter drive-through" sign revisited: A cadaveric study exploring its real mechanism based on the individual posterolateral structure of knee joints. Arch. Orthop. Trauma Surg. 2014, 134, 1745-1751. [CrossRef] [PubMed]

9. Johannsen, A.M.; Civitarese, D.M.; Padalecki, J.R.; Goldsmith, M.T.; Wijdicks, C.A.; Laprade, R.F. Qualitative and quantitative anatomic analysis of the posterior root attachments of the medial and lateral menisci. Am. J. Sports Med. 2012, 40, $2342-2347$. [CrossRef] [PubMed]

10. Nha, K.W.; Bae, J.H.; Kwon, J.H.; Kim, J.G.; Jo, D.Y.; Lim, H.C. Arthroscopic posteromedial drive-through test in posterior cruciate ligament insufficiency: A new diagnostic test. Knee Surg. Sport Traumatol. Arthrosc. 2015, 23, 1113-1118. [CrossRef] [PubMed]

11. Yang, G.Y.; Guo, H.L.; Li, T.; Shang, H.B.; Zhao, Y.F.; Shi, Y.Y. The medial compartment and patellofemoral joint degenerate more severely in early stage knee osteoarthritis: A cross-sectional study. Eur. Rev. Med. Pharmacol. Sci. 2020, 24, 9815-9823. [CrossRef] [PubMed]

12. Årøen, A.; Verdonk, P. Posterior cruciate ligament, exploring the unknown. Knee Surg. Sport Traumatol. Arthrosc. 2013, 21, 996-997. [CrossRef] [PubMed]

13. Ringler, M.D.; Shotts, E.E.; Collins, M.S.; Howe, B.M. Intra-articular pathology associated with isolated posterior cruciate ligament injury on MRI. Skelet. Radiol. 2016, 45, 1695-1703. [CrossRef] [PubMed]

14. Ahn, S.; Lee, Y.S.; Song, Y.D.; Chang, C.B.; Kang, S.B.; Choi, Y.S. Does surgical reconstruction produce better stability than conservative treatment in the isolated PCL injuries? Arch. Orthop. Trauma Surg. 2016, 136, 811-819. [CrossRef] [PubMed]

15. Wang, S.H.; Chien, W.C.; Chung, C.H.; Wang, Y.C.; Lin, L.C.; Pan, R.Y. Long-term results of posterior cruciate ligament tear with or without reconstruction: A nationwide, population-based cohort study. PLoS ONE 2018, 13, e0205118. [CrossRef] [PubMed] 